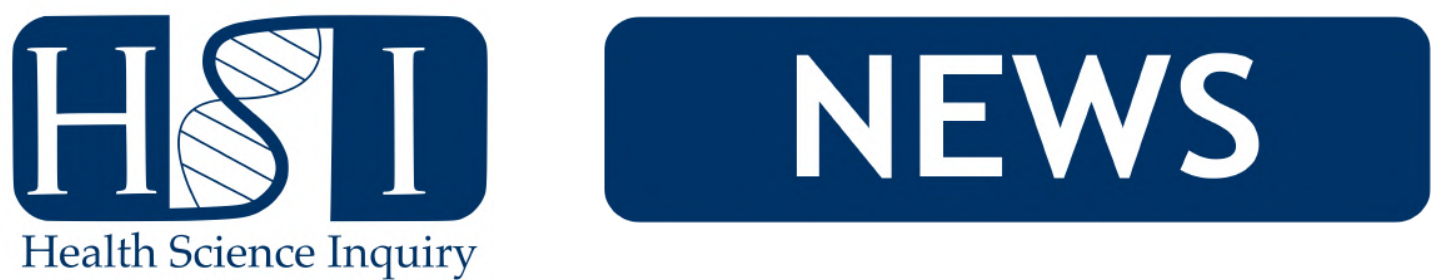

\title{
Epigenetics to the Rescue: Promising Potential of Epigenetic Therapies in Future Medicine
}

\section{Daniel Robinson}

The optimization of CRISPR technology to edit and correct errors in DNA is playing a pivotal role in developing new therapeutics which could soon cure genetic disease. However, genetic errors are not the only basis leading to disease. Such is the case when typical gene expression becomes aberrant, which in turn disrupts cellular and tissue function and culminates in disease. This specific regulation of gene expression beyond the actual DNA sequence is known as epigenetics. Broadly defined, this is the interaction of proteins and regulatory complexes with DNA which control whether a gene will be expressed or repressed, in turn providing cells specific functions. This differential regulation of genes allows cells which share a same DNA sequence within an organism to adopt different roles - such as the different functions of white blood cells versus skin cells - and providing different abilities to cell populations, such as the regenerative potential of liver cells. The ability to correct epigenetic dysfunction is a promising approach that could offer therapeutic value against various diseases, as is reviewed [1].

Muscle stem cells are a particularly attractive system for epigenetic studies given their abundance. Further, the role of muscle stem cells in regenerating damaged muscle is quite well understood - when skeletal muscle is damaged through exercise or disease, dormant muscle stem cells become activated, expand their population, then differentiate to repair damaged muscle (summarized in Figure 1). Thus, muscle stem cells are an attractive system to study how epigenetic changes confer regenerative potential to this population. In fact, researchers are now starting to see that epigenetics play a critical role in regulating these cell state changes.

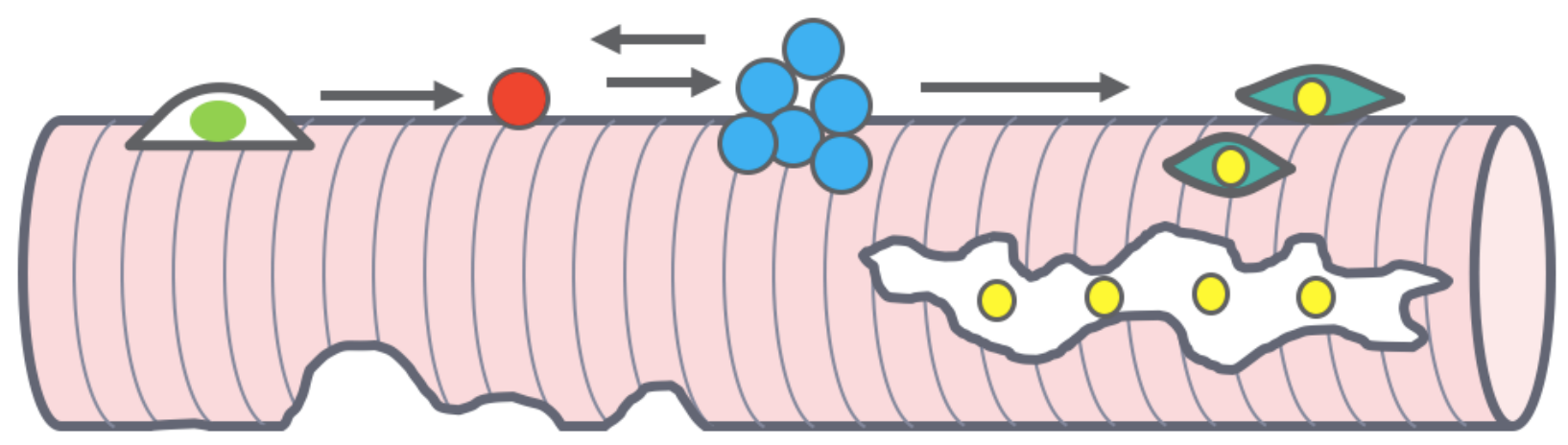

Figure 1: During muscle regeneration, dormant muscle stem cells (green) become activated (red), proliferate to expand its population (blue), then differentiate (yellow) to repair the damaged muscle fiber. These processes are regulated through dynamic changes within the epigenetic landscape.

At the Ottawa Hospital Research Institute's Sprott Center for Stem Cell Research, senior scientist Dr. Jeffrey Dilworth studies epigenetic implications in muscle stem cells. To shed some light on the subject, Dr. Dilworth explains that his research focus is to "understand how epigenetic mechanisms control cell state decisions made by muscle stem cells during development and regeneration, and how this information is used to regulate the regenerative potential of muscle stem cells". Dr. Dilworth believes his research will help clarify specifically how epigenetic changes regulate the regenerative potential of muscle stem cells in forming new muscle fibers. 
Current research has demonstrated that there are epigenetic differences of muscle stem cells during their regenerative process to fix damaged muscle fibers. Dr. Dilworth explains that dormant muscle stem cells have "condensed chromatin that [...] helps keep the cells resistant to DNA damage over time." When muscle damage is sustained and it becomes time to repair muscle, the dormant muscle stem cells become activated and start proliferating to expand the pool of cells required to regenerate skeletal muscle. At the epigenetic level, these proliferating muscle stem cells are "characterized by an opening of chromatin which allows higher expression of genes required for these processes to occur." Once a sufficient population of muscle progenitor cells is produced, another epigenetic change occurs which suppresses proliferative genes required for population expansion, and allows expression of new genes required for muscle function.

The importance of understanding epigenetic regulation in healthy muscle stem cells helps researchers understand their regenerative potential, and how this may be affected in diseased conditions. Dr. Dilworth explains that "[other research] groups have shown that in muscle wasting diseases, one contributing factor to loss of muscle is the inability of muscle stem cells to properly transition between cell states to help regeneration." The ability to correct epigenetic dysregulation in muscle wasting disease could help normalize muscle stem cell function and promote muscle strengthening. In theory, this will reduce the severity and progression of muscle wasting disease.

Fortunately, the clinical use of therapeutic agents to correct epigenetic states and return regenerative potential to muscle stem cells may be closer than expected. There are already some epigenetic drugs in clinical trials to treat muscle wasting diseases such as Duchenne Muscular Dystrophy. These histone deacetylase inhibitors, or HDACs which are reviewed here $[2,3]$ have shown positive results in animal models, but more research is required to see how this will translate into patients to improve muscle function.

The importance of epigenetic regulation in controlling cell roles and states is exemplifying the importance of proper gene regulation to maintain healthy cellular and tissue functions. Of interest is the recent discovery of epigenetic modifying drugs which can correct epigenetic states in diseased cell populations. Although the complete therapeutic extent of these drugs remains to be fully understood, epigenetic modifying drugs could hold great therapeutic value to treat various diseases in the future.

\section{REFERENCES}

[1] Heerboth S, Lapinska K, Snyder N, Leary M, Rollinson S, Sarkar S. Use of epigenetic drugs in disease: an overview. Genetics \& epigenetics. 2014;6:GEG-S12270.

[2] Consalvi S, Saccone V, Giordani L, Minetti G, Mozzetta C, Puri PL. Histone deacetylase inhibitors in the treatment of muscular dystrophies: epigenetic drugs for genetic diseases. Molecular medicine. 2011;17(5-6):457-465.

[3] Consalvi S, Saccone V, Mozzetta C. Histone deacetylase inhibitors: a potential epigenetic treatment for Duchenne muscular dystrophy. Epigenomics. 2014;6(5):547-560.

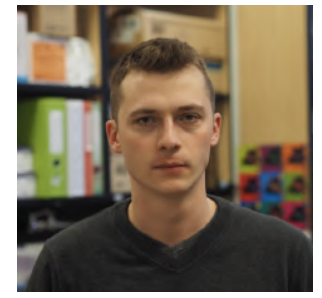

Daniel received his B.Sc. in Biochemistry at the University of Manitoba in 2015, where he worked in various labs ranging from synthetic organic chemistry to studying protein dynamics. He is currently a $\mathrm{PhD}$ candidate at the University of Ottawa, where he studies gene mechanisms important for muscle stem cell regenerative potential. During his free time, Daniel likes to stay active by jogging, cycling, and going to the gym. 